\title{
Therapeutic Benefits of Caffeine in Oral Submucous Fibrosis
}

\author{
Namrata Sengupta ${ }^{1}$, Sachin C Sarode ${ }^{2}$, Gargi S Sarode ${ }^{3}$, Urmi Ghone ${ }^{4}$, Shankargouda Patil ${ }^{5}$
}

World Journal of Dentistry (2021): 10.5005/jp-journals-10015-1835

Oral submucous fibrosis (OSMF) is a chronic condition associated with tissue fibrosis and often recognized as a precursor to oral cancer. It is usually characterized by chronic inflammation and abnormal collagen deposition leading to excessive fibrosis of the oral mucosal tissues. ${ }^{1}$ Burning sensation, pain, dry mouth, and trismus are also the other accompanying features of OSMF. ${ }^{1}$ Oral submucous fibrosis is mainly a disease of the Indian subcontinent where the people with low socioeconomic status are affected the most due to the habit of betel nut chewing. ${ }^{2}$

Betel nut, the most accepted causative factor of OSMF, contributes to various alterations in collagen metabolism. ${ }^{3,4}$ The alkaloids in betel nut, such as arecoline and arecaidine, trigger the fibroblasts to produce collagen. ${ }^{5}$ This collagen production is unrestricted when compared to collagen production by normal cells. ${ }^{6}$ Addition of slaked lime to betel nut augments the stimulation of fibroblasts leading to their excessive proliferation and collagen production. ${ }^{7}$ A part from abundant collagen synthesis, the alkaloids of betel nut also help in cross-linking of the collagen peptide chains. ${ }^{8}$ Thus, the excess collagen produced now becomes resistant to degradation by enzymes like collagenases. The fibroblasts themselves participate in collagen break-down by phagocytosis but in OSMF patients fibroblasts are remarkably deficient in collagen phagocytosis. ${ }^{5}$ Thus, once initiated, the collagen metabolism dysregulation continues in these patients. This dysregulation is further amplified by a cytokine, TGF- $\beta$ which is a product of the local inflammatory reaction. ${ }^{5}$ It activates procollagen genes and significantly stimulates collagen synthesis. It also increases the level of the enzyme lysyl oxidase (LOX) which favors cross-linking of the collagen fibers and their reduced degradation. ${ }^{5}$

The dysregulation of collagen metabolism has been the hotspot of many studies on OSMF which has a significant malignant transformation rate. ${ }^{5,9,10}$ Advances have been made to develop appropriate therapeutic aids for its management. Local injections of corticosteroids along with hyaluronidase or placental extract have been employed in its management strategies. ${ }^{11}$ Corticosteroids are thought to suppress the inflammatory reaction. This reduces fibrosis by decreasing fibroblastic proliferation. ${ }^{11}$ Many natural products have also been explored to improve the dysregulation process in OSMF. ${ }^{12}$

Caffeine is one such naturally occurring compound that has a wide range of pharmacological activities. In recent studies, it has been found that caffeine exhibits antifibrotic effects in the liver. ${ }^{13}$ It does so by downregulating collagen expression as well as deposition. Caffeine also blocks the expression of TGF- $\beta$, a profibrotic cytokine. Such a potential effect of caffeine was also examined on lung fibrosis. ${ }^{14}$ The study reported that caffeine showed its antifibrotic effect in the lungs by acting on two different types of cells, i.e., the epithelial cells and the fibroblasts. ${ }^{14}$ Caffeine prevented the activation of TGF- $\beta$ by the lung epithelial cells in a concentration-dependent manner.
${ }^{1-4}$ Department of Oral Pathology and Microbiology, Dr DY Patil Dental College and Hospital, Dr DY Patil Vidyapeeth, Pune, Maharashtra, India

${ }^{5}$ Department of Maxillofacial Surgery and Diagnostic Sciences, Division of Oral Pathology, College of Dentistry, Jazan University, Jazan, Kingdom of Saudi Arabia

Corresponding Author: Namrata Sengupta, Department of Oral Pathology and Microbiology, Dr DY Patil Dental College and Hospital, Dr DY Patil Vidyapeeth, Pune, Maharashtra, India, Phone: +91 8116807494, e-mail: dr.namrata.sengupta@gmail.com

How to cite this article: Sengupta N, Sarode SC, Sarode GS, et al. Therapeutic Benefits of Caffeine in Oral Submucous Fibrosis. World J Dent 2021;12(4):263-264.

Source of support: Nil

Conflict of interest: None

It also nullified the profibrotic response to TGF- $\beta$ within the lung fibroblasts. Further investigations of the study included observation of actions of caffeine on TGF- $\beta$-induced gene expression. TGF- $\beta$ upregulated the expressions of PAI1, TGFB1, and ACTA2 in idiopathic pulmonary fibrosis (IPF) fibroblasts, and caffeine was able to abrogate these responses as well. An upsurge of expression of $a$-smooth muscle actin (a-SMA) within fibroblasts is an important indicator of fibroblast-myofibroblast transdifferentiation. Caffeine was also found to be a cause of interruption of such a fibrogenic process. Not only in the liver and lungs, but caffeine has also been shown to reduce collagen synthesis in human cultured skin fibroblasts. ${ }^{15}$

Caffeine can be employed as a therapeutic intervention in the treatment of OSMF. Oral submucous fibrosis mainly affects individuals of low socioeconomic status, who have a habit of drinking tea 2-3 times a day. Instead of tea, coffee can be consumed twice or thrice a day to get the beneficial effects of caffeine. The disadvantage of coffee consumption is that caffeine will be first absorbed in systemic circulation and then reach the oral mucosa to show its effect. To amplify the local effect of caffeine, coffee beans can be chewed instead of areca nut/betel nut. These beans are rich sources of caffeine as well as antioxidants and are edible. Eating coffee allows the active compounds of the bean to get absorbed through the oral mucous membrane. Such homemade interventions might improve the conditions of OSMF patients but the financial aspect of such modifications should also be kept in mind. Coffee is costlier than tea and these remedies might be difficult to afford in a routine for OSMF patients belonging to low economic class. Hence, caffeine-rich mouth gels can be made available in the market. If possible, coffee beans packaged in small quantities can be marketed. Caffeine-rich chewing gums can also be employed in such modified interventions. 
The potential antifibrotic effect of caffeine in OSMF should be in the limelight of future studies. TGF- $\beta$ is the key player in the disease progression of OSMF. It controls the expression of a-SMA in myofibroblasts. Hence, the effect of caffeine on oral epithelial cells and fibroblasts will develop promising research hotspots and open gateways to potential therapeutic interventions in the management of OSMF. Small lifestyle modifications might show prospective and improved changes in the treatment approaches of OSMF.

\section{References}

1. Shih YH, Wang TH, Shieh TM, et al. Oral submucous fibrosis: a review on etiopathogenesis, diagnosis, and therapy. Int J Mol Sci 2019;20(12):2940. DOI: 10.3390/ijms20122940.

2. Ahmad MS, Ali SA, Ali AS, et al. Epidemiological and etiological study of oral submucous fibrosis among gutkha chewers of Patna, Bihar, India. J Indian Soc Pedod Prev Dent 2006;24(2):84-89. DOI: 10.4103/0970-4388.26022.

3. Aziz SR. Oral submucous fibrosis: case report and review of diagnosis and treatment. J Oral Maxillofac Surg 2008;66(11):2386-2389. DOI: 10.1016/j.joms.2008.06.064.

4. Arakeri G, Brennan PA. Oral submucous fibrosis: an overview of the aetiology, pathogenesis, classification, and principles of management. Br J Oral Maxillofac Surg 2013;51(7):587-593. DOI: 10.1016/j.bjoms.2012.08.014

5. Arakeri G, Rai KK, Hunasgi S, et al. Oral submucous fibrosis: an update on current theories of pathogenesis. J Oral Pathol Med 2017;46(6):406-412. DOI: 10.1111/jop.12581.
6. Liu CM, Liao YW, Hsieh PL, et al. miR-1246 as a therapeutic target in oral submucosa fibrosis pathogenesis. J Formos Med Assoc 2019;118(7):1093-1098. DOI: 10.1016/j.jfma.2019.02.014.

7. Angadi PV, Rao SS. Areca nut in pathogenesis of oral submucous fibrosis: revisited. Oral Maxillofac Surg 2011;15(1):1-9. DOI: 10.1007/ s10006-010-0219-8.

8. Prabhu RV, Prabhu V, Chatra L, et al. Areca nut and its role in oral submucous fibrosis. J Clin Exp Dent 2014;6(5):e569-e575. DOI: 10.4317/jced.51318.

9. Shen YW, Shih YH, Fuh LJ, et al. Oral submucous fibrosis: a review on biomarkers, pathogenic mechanisms, and treatments. Int J Mol Sci 2020;21(19):7231. DOI: 10.3390/ijms21197231.

10. Peng $\mathrm{Q}, \mathrm{LiH}, \mathrm{Chen} \mathrm{J}$, et al. Oral submucous fibrosis in Asian countries. J Oral Pathol Med 2020;49(4):294-304. DOI: 10.1111/jop.12924.

11. James L, Shetty A, Rishi D, et al. Management of oral submucous fibrosis with injection of hyaluronidase and dexamethasone in grade III oral submucous fibrosis: a retrospective study. J Int Oral Health 2015;7(8):82-85.

12. Rajesh Kashyap R, Shanker Kashyap R. Herbal derivatives in the management of mouth opening in oral submucous fibrosis-a network meta-analysis. Oral Dis 2020. DOI: 10.1111/odi.13544.

13. Modi AA, Feld JJ, Park Y, et al. Increased caffeine consumption is associated with reduced hepatic fibrosis. Hepatology 2010;51(1):201209. DOI: 10.1002/hep.23279.

14. Tatler AL, Barnes J, Habgood A, et al. Caffeine inhibits TGF $\beta$ activation in epithelial cells, interrupts fibroblast responses to TGF $\beta$, and reduces established fibrosis in ex vivo precision-cut lung slices. Thorax 2016;71(6):565-567. DOI: 10.1136/thoraxjnl-2015-208215.

15. Donejko M, Przylipiak A, Rysiak E, et al. Influence of caffeine and hyaluronic acid on collagen biosynthesis in human skin fibroblasts. Drug Des Devel Ther 2014;8:1923-1928. DOI: 10.2147/DDDT.S69791. 